\title{
Management of Tibial Bony Defect with Metal Block in Primary Total Knee Replacement Arthroplasty
}

\author{
Seung-Wook Baek, MD, Chul-Woong Kim, MD, and Choong Hyeok Choi, MD \\ Department of Orthopaedic Surgery, Hanyang University College of Medicine, Seoul, Korea
}

Purpose: To analyze minimum 2-year clinical and radiological follow-up results of primary total knee replacement arthroplasty (TKRA) with metal block augmentation for tibial bony defect.

Materials and Methods: We analyzed 67 cases (52 patients) of primary TKRA with metal block augmentation for tibial bony defects from March 1999 and March 2008. Clinical results were evaluated using the Knee Society clinical rating system and the Western Ontario and McMaster University (WOMAC) score. Radiologic results were evaluated using the Knee Society roentgenographic evaluation system.

Results: The mean knee score and function score improved from 42.0 and 45.6 preoperatively to 94.5 and 85.4 postoperatively. At last followup, the mean WOMAC score was 16.8. The incidence of radiolucent lines was 10\% (7 cases) during the follow-up period, but there was no case of progression. There were no statistically significant differences between the groups divided according to the block size (below $5 \mathrm{~mm}$ and over $8 \mathrm{~mm}$ ) and between the stem and no-stem groups for all parameters.

Conclusions: Primary TKRA with a metal block produced satisfactory results for the minimum 2-year follow-up and can be considered as a simple and effective method for the treatment of tibial bony defect in primary TKRA.

Keywords: Total knee replacement arthroplasty, Bony defect, Metal block

\section{Introduction}

Tibial bony defect is commonly encountered during total knee replacement arthroplasty (TKRA) for osteoarthritis. It has been associated with angular position and stability of the implants after TKRA. Therefore, augmentation of bony defects is crucial to the maintenance of implant stability and alignment and longevity of TKRA $^{1,2)}$.

Tibial bony defect has been managed with bone cementing, insertion of a thick polyethylene implant after bone resec-

Received August 10, 2011; Revised (1st) September 20, 2011;

(2nd) February 20, 2012; (3rd) March 20, 2012;

Accepted January 15, 2013

Correspondence to: Choong Hyeok Choi, MD

Department of Orthopaedic Surgery, Hanyang University College of Medicine, 222 Wangsimni-ro, Seongdong-gu, Seoul 133-791, Korea Tel: +82-2-2290-8485, Fax: +82-2-2299-3774

E-mail: chhchoi@hanyang.ac.kr

This is an Open Access article distributed under the terms of the Creative Commons Attribution Non-Commercial License (http://creativecommons.org/licenses/by-nc/3.0/) which permits unrestricted non-commercial use, distribution, and reproduction in any medium, provided the original work is properly cited. tion down to bone defect, or bone grafting ${ }^{3-9)}$. Some recent studies have introduced metal wedge or block augmentation methods ${ }^{10-12)}$. However, there are still controversies over the advantages and disadvantages of these methods. In particular, there is a paucity in the literature on the results of primary TKRA with metal block augmentation.

In the current study, we analyzed clinical and radiographic results of primary TKRA with metal block augmentation for tibial bony defect with a minimum 2-year follow-up. In addition, the influence of the block thickness and the use of a stem on the results was also investigated.

\section{Materials and Methods}

A total of 910 primary TKRAs were performed on 593 patients at our institution between March 1999 and March 2008 and metal block augmentation for tibial bony defect was carried out in 92 cases ( 72 patients) during TKRA. Of these 92 cases, the results in 67 cases (52 patients) available for $\geq 2$ years of follow-up were analyzed in this study. The mean age of the patients ranged from 45 to 82 years with a mean age of 64.8 years. There were 47 females and 5 males. The mean follow-up period was 5.3 years 


\section{Baek et al. Management of Tibial Bony Defect with Metal Block in Primary Total Knee Replacement Arthroplasty}

(range, 2 to 10.7 years). The cause of surgery was osteoarthritis in 54 cases and rheumatoid arthritis in 13 cases. The prosthesis used was Scorpio (Stryker, Mahway, NJ, USA) in 59 cases and Nexgen LPS (Zimmer, Warsaw, IN, USA) in 8 cases. In all cases, a posterior cruciate ligament substituting type of prosthesis was fixated with bone cement.

After tibial bone resection at a site $10 \mathrm{~mm}$ distal to the lateral plateau, metal block augmentation was performed for a noncontained defect in the medial cut surface, if it was $\geq 3 \mathrm{~mm}$ deep from the inferior surface of the tibial component and involved $\geq 1 / 3$ of the medial compartment. The deficient and sclerotic areas in the medial compartment were prepared in rectangular shape to approximate the metal block size and thickness $(4,5,8$, or $10 \mathrm{~mm}$ ). In the 67 cases, a 4 or $5 \mathrm{~mm}$ metal block was used in 29 cases, a 8 or $10 \mathrm{~mm}$ block in 35 cases, and double blocks in 3 cases.

A stem was used in all cases with an 8 or $10 \mathrm{~mm}$ metal block or double blocks, and in some cases with a 4 or $5 \mathrm{~mm}$ block if implant construct stability was considered insufficient during the varus-valgus stress test and flexion-extension test for soft-tissue balancing with use of a trial prosthesis and a metal block or due to poor bone quality of the proximal tibia that could result in implant subsidence. Therefore, a stem was used in 42 cases (63\%): 33 cases with an 8 or $10 \mathrm{~mm}$ metal block, 3 cases with double blocks, and 6 of the 29 cases with a $5 \mathrm{~mm}$ metal block. The length of the stem was $\geq 80 \mathrm{~mm}$ in 33 cases (79\%) and $\leq 70 \mathrm{~mm}$ in 9 cases (21\%).

Clinical assessments were done by an independent orthopedic surgeon based on the pre- and postoperative range of motion (ROM), the Knee Society clinical score, and the Western Ontario and McMaster University (WOMAC) score at the last follow-up.

On the radiographic evaluation, femorotibial alignment was assessed on the pre- and postoperative and last follow-up standing radiographs, and the presence of periprosthetic radiolucency and component loosening was evaluated on the anteroposterior (AP) radiographs taken using fluoroscopy, lateral views focused on the femoral component and tibial component each, and axial views. The Knee Society roentgenographic evaluation system ${ }^{13)}$ was used for the assessment of the periprosthetic radiolucency and loosening. Radiographic measurements were performed twice each by two knee surgeons. Inter- and intraobserver reliability was evaluated by kappa values. Statistical analysis of the results was done using SPSS ver. 17.0 (SPSS Inc., Chicago, IL, USA). Statistical significance of the influence of the block thickness and the use of a stem on the clinical results was assessed using the independent $\mathrm{t}$-test and on the radiographic results using the
Fisher's exact test with a significance level set at 0.05 .

\section{Results}

\section{Clinical Evaluation}

Regarding the ROM, the mean flexion contracture was corrected from $12.0^{\circ}$ (range, $0^{\circ}$ to $40^{\circ}$ ) preoperatively to $1.1^{\circ}$ (range, $0^{\circ}$ to $10^{\circ}$ ) at the last follow-up, and the mean maximum flexion was $122.7^{\circ}$ (range, $75^{\circ}$ to $150^{\circ}$ ) preoperatively and $123.7^{\circ}$ (range, $100^{\circ}$ to $140^{\circ}$ ) at the last follow-up.

The mean knee score improved from 42.0 points (range, 3 to 75 points) preoperatively to 94.5 points (range, 79 to 100 points) at the last follow-up. The mean function score increased from 45.6 points (range, 5 to 75 points) preoperatively to 85.4 points (range, 60 to 100 points) at the last follow-up. The mean knee score and function score in the $\leq 5 \mathrm{~mm}$ metal block group were 95.8 points (range, 87 to 100 points) and 87.1 points (range, 60 to 100 points), respectively, and in the $\geq 8 \mathrm{~mm}$ metal block group were 92.8 points (range, 79 to 100 points) and 82.8 points (range, 60 to 100 points), respectively, showing no statistically significant intergroup difference $(\mathrm{p}=0.323, \mathrm{p}=0.274)$. The mean knee score and function in the no-stem group were 94.6 points (range, 87 to 100 points) and 86.9 points (range, 60 to 100 points), respectively, and in the stem group were 94.2 points (range, 79 to 100 points) and 84.5 points (range, 60 to 100 points), respectively, indicating no significant intergroup difference $(\mathrm{p}=0.850, \mathrm{p}=0.362)$.

At the last follow-up, the mean WOMAC score was 16.8 points (range, 4 to 39 points) with the mean pain score 3.0 points (range, 0 to 6 points), mean symptom score 0.7 points (range, 0 to 2 points), and mean physical function score 13 points (range, 4 to 31 points). The mean WOMAC score was lower in the $\leq 5 \mathrm{~mm}$ metal block group (15.8 points; range, 9 to 36 points) than in the $\geq 8 \mathrm{~mm}$ metal block group (17.5; range, 4 to 39 points), but the difference was not statistically significant $(\mathrm{p}=0.282)$. The mean WOMAC score was not notably different between the no-stem group (15.3 points; range, 9 to 27 points) and the stem group (17.7 points; range, 4 to 39 points; $\mathrm{p}=0.115$ ).

\section{Radiographic Evaluation}

Preoperative radiographs showed varus deformity in 66 cases and valgus deformity in 1 case. The mean femorotibial alignment was corrected from a varus of $10.8^{\circ}$ (range, $-25.5^{\circ}$ to $4.5^{\circ}$ ) preoperatively to a valgus of $5.0^{\circ}$ (range, $1.3^{\circ}$ to $12.2^{\circ}$ ) postoperatively in the medial metal block augmentation group and from a valgus of $18.3^{\circ}$ preoperatively to a valgus of $4.1^{\circ}$ postoperatively in the lateral metal block augmentation group. 

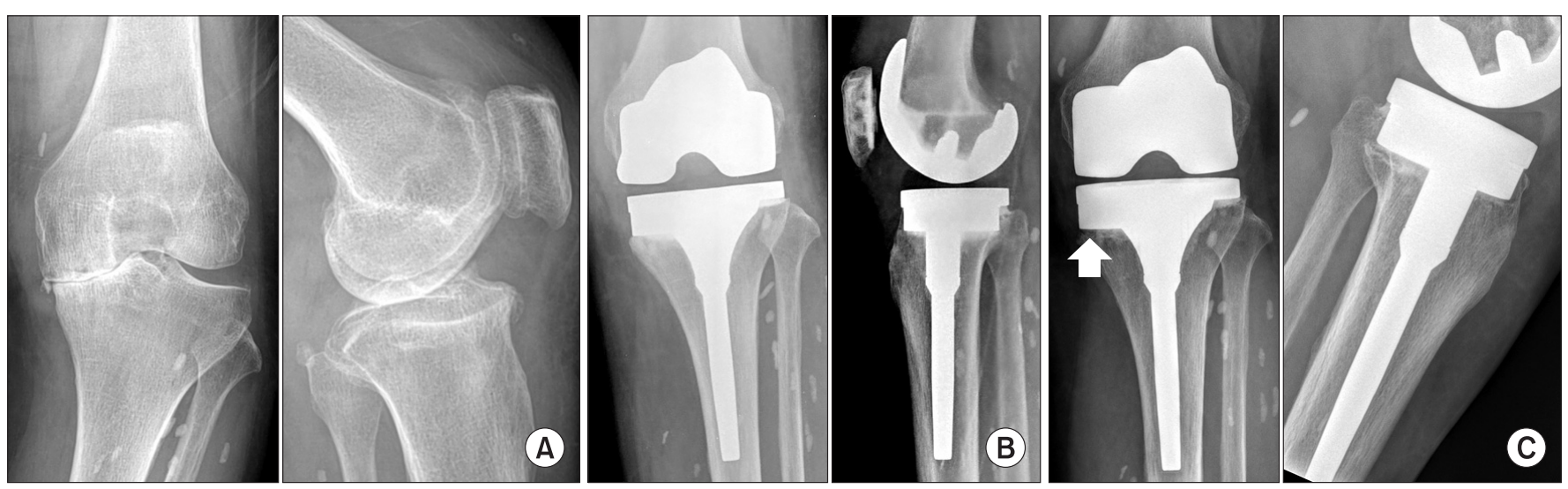

Fig. 1. A 63-year-old female. (A) Preoperative anteroposterior (AP) and lateral views showed osteoarthritis with a bony defect in the medial tibial plateau and a $12^{\circ}$ varus deformity of the right knee joint. (B) We performed total knee replacement arthroplasty with a metal block. Immediate postoperative AP and lateral views showed correction to $6^{\circ}$ valgus tibio-femoral alignment. (C) The less than 1 mm radiolucent area between the cement and bone (white arrow) was noted on the medial side on the AP and lateral fluoroscopic images at 4 years follow-up.

At the last follow-up, radiolucency related to bone resorption was observed immediately below the tibial metal block in 7 cases $(10 \%)$ in the medial metal block augmentation group. The radiolucent lines in all of these cases were found in zone 1 according to the Knee Society roentgenographic evaluation system. In addition, the radiolucency score was relatively low: 1 point in 4 cases in the $\leq 5 \mathrm{~mm}$ metal block group, and 2 points in 1 case and 1 point in 2 cases in the $\geq 8 \mathrm{~mm}$ metal block group. Accordingly, regular follow-up evaluations were not considered necessary and signs of component loosening and displacement were not noted (Fig. 1).

The reliability of assessments of radiolucency and loosening performed twice by each of the two knee surgeons was high. The kappa values for intraobserver reliability were 0.817 and 0.817 for each assessment session and the values for interobserver reliability were $0.817,0.784$ for each session. There were no significant intergroup differences in the appearance of radiolucent lines: radiolucency was present in 4 cases (6\%) in the $\leq 5 \mathrm{~mm}$ metal block group and in 3 cases (4\%) in the $\geq 8 \mathrm{~mm}$ metal block group ( $\mathrm{p}=0.691$ ); and in 3 cases ( $4 \%$ ) in the no-stem group and in 4 cases (6\%) in the stem group ( $\mathrm{p}=1.00)$.

\section{Complications}

Complications such as deep infection, wound necrosis, knee joint stiffness, displacement of the metal block, breakdown of the lower portion of the metal block or varus collapse and fracture around metal block were not observed. Adjuvant surgery or reoperation due to pain or functional impairment was not necessary in any of the cases. In 9 cases, 40 to 45 points of mild pain according to the Knee Society scoring system was noted, but it did not disrupt normal daily living activities. There were no cases with $\leq 30$ points of moderate pain. Neither irritation of the medial collateral ligament nor tenderness due to the metal block was reported in any of the cases.

\section{Discussion}

Tibial bony defects are occasionally encountered during TKRA. In general, if $\geq 40 \%$ of the contact surface between the bone and the implant is not supported by the host bone, augmentation for bony defect is performed to maintain implant stability ${ }^{11}$. The currently available management options for bony defect include the insertion of a thicker polyethylene component after bone resection that extends distal to the deficient area, bone cementing with or without screw reinforcement, bone grafting, metal wedge or block augmentation, and the use of a custom-made component ${ }^{3-12)}$. A more distal resection down to tibial deficiency can compromise the strength of the cancellous bone in the proximal tibia and implant stability. In addition, a smaller tibial component should be used after more distal tibial resection because of the conical shape of proximal tibia. In this situation, mismatching of tibial and femoral component sizing can be a concern ${ }^{14)}$. Therefore, in this study, tibial resection was performed at a site $10 \mathrm{~mm}$ distal to the lateral plateau irrespective of the extent of tibial deficiency, which was followed by a bony defect management procedure. Bone cement alone can be considered sufficient in knees with $\leq 5 \mathrm{~mm}$ bone loss, whereas screw reinforcement with reduced use of bone cement has been suggested as a proper solution in the presence of $\geq 5 \mathrm{~mm}$ tibial bony defect ${ }^{8,15)}$. We have managed $\leq 2 \mathrm{~mm}$ non-contained bone 
defects that involve $<1 / 3$ of one compartment with bone cement only. However, for larger bone defects, a rectangular metal block has been used for augmentation, considering that the use of massive amount of bone cement can lead to breakdown of the cement or atrophy and wedge-shaped bone defects cannot be sufficiently managed with bone cement. Bone grafting for tibial bony defect, first introduced by Windsor et al. ${ }^{16)}$, can be performed using autogenous bone grafts obtained during primary TKRA. This method promotes anatomical recovery of bone quality and provides biological and biomechanical benefits to young patients who may expect a revision TKRA in the future. The failure rate of bone grafting at 6.8 years after TKRA was reported as $3 \%$ in the study by Watanabe et al. ${ }^{17}$. Ahmed et al. ${ }^{18)}$ reported there was no case of failure during 10 years of follow-up period. We have used autogenous bone grafts obtained during tibial resection for contained defects and attempted to restore bone quality with the use of autogenous or allogenic bone grafts in young patients with non-contained defects. However, in order to promote bone union for restoration of bone quality after grafting, the sclerotic or abnormal cancellous bone should be completely removed until the normal cancellous bone is exposed. During this procedure, bony defect area would be made larger. That means an originally $3 \mathrm{~mm}$ deep bony defect would be treated as a more than $10 \mathrm{~mm}$ deep defect during TKRA. In such a case, autogenous bone grafts obtained from primary TKRA may not be sufficient for grafting and the use of a stem may be required. In the absence of prepared allogenic grafts or patients' consent on the use of allografts or available stems, bone grafting can be difficult or impossible to perform. Even in the circumstances where allogenic grafts can be transplanted, it is technically difficult to fixate the grafts in the non-contained deficient area, operation time should be extended, and the risk of transmitted diseases increases in rare case, though. Laskin ${ }^{19)}$ reported 33\% failure rate at 5 years after TKRA using autogenous bone grafts. In addition, the incidence and timing of bone resorption and maintenance of biomechanical properties remain controversial. Although we did not use a metal block for every tibial bony defect, if extensive bone resection was expected necessary to expose the unaffected cancellous bone for proper bone grafting, we carried out metal block augmentation after partial removal of the sclerotic bone and additional bone drilling on the bone bed.

Tibial bony defect management has been remarkably improved with the use of metal augments. Rectangular or wedge-shaped tibial augments can be fixated with bone cement or screws and useful for $\leq 20 \mathrm{~mm}$ bone defects. Metal augments can be customized in shape intraoperatively without significant increases in operation time, are economical and applicable to various operations ${ }^{1}$, and provide biomechanical implant stability for weight-bearing and joint movements ${ }^{20,211}$. However, metal augmentation does not lead to anatomical restoration of bone quality and may not be recommended in young patients because normal bone should be included in resection and the resultant extensive bone loss may impair revision TKRA. The durability of metal augments has been questioned by some authors including Brand et al. ${ }^{22)}$ who reported the incidence of radiolucent lines was $25 \%$ at 3.5 years after TKRA using metal wedges. Compared to autogenous bone grafting, the procedure requires additional expense for the metal augments. In addition, protrusion of the augments may cause pain in the collateral ligaments ${ }^{2}$. In this study, tibial resection was performed at a site $10 \mathrm{~mm}$ distal to the lateral plateau and a rectangular metal block was inserted if a non-contained defect involved $\geq 3 \mathrm{~mm}$ from the inferior surface of the tibial component and $\geq 1 / 3$ of the medial compartment. The deficient area in the medial compartment and the sclerotic area were removed in a rectangular shape that approximates the thickness of the prepared block $(4,5,8$, or $10 \mathrm{~mm})$ determined according to the implant size. Care was taken to choose a smaller block than the tibial component to prevent protruding metal augments from irritating the medial collateral ligament at the tibial resection area due to the conical shape of the proximal tibia. There was no case of postoperative irritation or pain related to the protrusion of metal augments.

Stems provide support for implants with metal augments, fixation stability, distribution of shear stress, and reduction in bone cement stress ${ }^{1,20,23,24)}$. In this study, stems were used to ensure implant stability in cases with an 8 or $10 \mathrm{~mm}$ metal block or double blocks. Stems were also additionally implanted to distribute stress in the proximal tibia in case with poor bone quality in the proximal tibia or in cases with a 4 or $5 \mathrm{~mm}$ augment, if stability was considered insufficient when tested with a trial prosthesis augmented with a metal block. The diameter and length of a stem are negatively correlated with the incidence of implant displacement. A thick and long stem is effective for obtaining rigid fixation without any increase in stress shielding effect, but it may cause stem tip pain that affects postoperative outcome $^{25}$. In this study, we determined the length and diameter of a stem according to the anatomical shape of the proximal tibia and the results of the varus-valgus stress and flexion-extension gap balance tests conducted with a trial prosthesis augmented with a metal block placed.

Metal augments can be either a block or wedge in shape and 
the benefits and pitfalls of these augments remain controversial. Fehring et al. ${ }^{26)}$ reported that blocks resulted in less deformation than wedges based on the analysis of strain distribution, tensile deformation rate, compression deformation rate, and shear deformation rate. Chen and Krackow $^{27)}$ reported that rectangular metal blocks placed on the surface of bone defects vertical to the loading direction were more effective in reducing shear force than metal wedges on the oblique surface of defects. Metal wedge augments allow for less bone resection than metal blocks. On the other hand, metal block augmentation is more stable from a biomechanical standpoint and technically easier to perform. For these reasons, we used rectangular augments in this study.

In this study, we evaluated the minimum 2-year followup results of primary TKRA with metal block augmentation. The clinical results were satisfactory. There was no case of component loosening. Non-progressive radiolucency related to bone resorption was observed around the augment in $10 \%$ of the cases. However, we think the short-term results should be confirmed by long-term follow-up. In addition, we believe the possibility of revision TKRA should be taken into consideration in determining metal block augmentation because the procedure does not involve restoration of bone stock and the remaining bone deficiency may complicate future surgery.

\section{Conclusions}

The clinical and radiographic results of primary TKRA with metal block augmentation were satisfactory in our at least 2-year follow-up. Although our results should be confirmed by longterm follow-up, metal block augmentation can be considered as a simple and effective method for the treatment of tibial bony defect in primary TKRA.

\section{Conflict of Interest}

No potential conflict of interest relevant to this article was reported.

\section{References}

1. Radnay CS, Scuderi GR. Management of bone loss: augments, cones, offset stems. Clin Orthop Relat Res. 2006;446: 83-92.

2. Cuckler JM. Bone loss in total knee arthroplasty: graft augment and options. J Arthroplasty. 2004;19(4 Suppl 1):56-8.

3. Harris AI, Poddar S, Gitelis S, Sheinkop MB, Rosenberg
AG. Arthroplasty with a composite of an allograft and a prosthesis for knees with severe deficiency of bone. J Bone Joint Surg Am. 1995;77:373-86.

4. Altchek D, Sculco TP, Rawlins B. Autogenous bone grafting for severe angular deformity in total knee arthroplasty. J Arthroplasty. 1989;4:151-5.

5. Elia EA, Lotke PA. Results of revision total knee arthroplasty associated with significant bone loss. Clin Orthop Relat Res. 1991;(271):114-21.

6. Engh GA, Ammeen D. Results of total knee arthroplasty with medial epicondylar osteotomy to correct varus deformity. Clin Orthop Relat Res. 1999;(367):141-8.

7. Fipp GJ. A bone grafting technique in reconstructive joint arthroplasty. J Arthroplasty. 1989;4:285-7.

8. Ritter MA, Keating EM, Faris PM. Screw and cement fixation of large defects in total knee arthroplasty: a sequel. J Arthroplasty. 1993;8:63-5.

9. van Loon CJ, Pluk C, de Waal Malefijt MC, de Kock M, Veth RP. The GSB total knee arthroplasty: a medium- and longterm follow-up and survival analysis. Arch Orthop Trauma Surg. 2001;121:26-30.

10. Lee JK, Choi $\mathrm{CH}$. Management of tibial bone defects with metal augmentation in primary total knee replacement: a minimum five-year review. J Bone Joint Surg Br. 2011;93: 1493-6.

11. Dennis DA. Repairing minor bone defects: augmentation \& autograft. Orthopedics. 1998;21:1036-8.

12. Rand JA. Modular augments in revision total knee arthroplasty. Orthop Clin North Am. 1998;29:347-53.

13. Ewald FC. The Knee Society total knee arthroplasty roentgenographic evaluation and scoring system. Clin Orthop Relat Res. 1989;(248):9-12.

14. Harada Y, Wevers HW, Cooke TD. Distribution of bone strength in the proximal tibia. J Arthroplasty. 1988;3:167-75.

15. Lee RW, Volz RG, Sheridan DC. The role of fixation and bone quality on the mechanical stability of tibial knee components. Clin Orthop Relat Res. 1991;(273):177-83.

16. Windsor RE, Insall JN, Sculco TP. Bone grafting of tibial defects in primary and revision total knee arthroplasty. Clin Orthop Relat Res. 1986;(205):132-7.

17. Watanabe W, Sato K, Itoi E. Autologous bone grafting without screw fixation for tibial defects in total knee arthroplasty. J Orthop Sci. 2001;6:481-6.

18. Ahmed I, Logan M, Alipour F, Dashti H, Hadden WA. Autogenous bone grafting of uncontained bony defects of tibia during total knee arthroplasty a 10 -year follow up. J 
Arthroplasty. 2008;23:744-50.

19. Laskin RS. Total knee arthroplasty in the presence of large bony defects of the tibia and marked knee instability. Clin Orthop Relat Res. 1989;(248):66-70.

20. Hoeffel DP, Rubash HE. Revision total knee arthroplasty: current rationale and techniques for femoral component revision. Clin Orthop Relat Res. 2000;(380):116-32.

21. Partington PF, Sawhney J, Rorabeck CH, Barrack RL, Moore J. Joint line restoration after revision total knee arthroplasty. Clin Orthop Relat Res. 1999;(367):165-71.

22. Brand MG, Daley RJ, Ewald FC, Scott RD. Tibial tray augmentation with modular metal wedges for tibial bone stock deficiency. Clin Orthop Relat Res. 1989;(248):71-9.

23. Murray PB, Rand JA, Hanssen AD. Cemented long-stem revision total knee arthroplasty. Clin Orthop Relat Res.
1994;(309):116-23.

24. Conditt MA, Parsley BS, Alexander JW, Doherty SD, Noble PC. The optimal strategy for stable tibial fixation in revision total knee arthroplasty. J Arthroplasty. 2004;19(7 Suppl 2):113-8.

25. Jazrawi LM, Bai B, Kummer FJ, Hiebert R, Stuchin SA. The effect of stem modularity and mode of fixation on tibial component stability in revision total knee arthroplasty. J Arthroplasty. 2001;16:759-67.

26. Fehring TK, Peindl RD, Humble RS, Harrow ME, Frick SL. Modular tibial augmentations in total knee arthroplasty. Clin Orthop Relat Res. 1996;(327):207-17.

27. Chen F, Krackow KA. Management of tibial defects in total knee arthroplasty: a biomechanical study. Clin Orthop Relat Res. 1994;(305):249-57. 